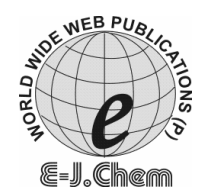

http://www.e-journals.net
ISSN: 0973-4945; CODEN ECJHAO

E-Journal of Chemistry 2010, 7(2), 636-640

\title{
Synthesis of Benzofuran Derivatives and their Evaluation of Antimicrobial Activity
}

\author{
D. R. HARISH KUMAR ${ }^{*}$ and M. D. KARVEKAR \\ Department of Pharmaceutical Chemistry, \\ Krupanidhi College of Pharmacy, Bangalore-560035, India. \\ drhkshs@yahoo.com
}

Received 29 August 2009; Accepted 20 October 2009

\begin{abstract}
Acetyl benzofuran (1) was synthesized by refluxing salicylaldehyde and dry chloroacetone in presence of potassium carbonate. The product formed was reacted with 2 -aminobenzothiazole with catalyst $p$-toluene sulphonic acid to form $N$-[(1Z)-1-(benzofuran-2-yl)ethylidene]1,3-benzothiazol-2-amine (2). In cold condition, Staudinger reaction was carried out for compound (2) with various acid chlorides in presence of triethylamine to synthesize 4-[1benzofuran-2-yl]-1-[1, 3-benzothiazol-2-yl]-4-methylazetidin-2-one (3a-3j). All the synthesized compounds were characterized on the basis of analytical data. The compounds were screened for their antimicrobial activities. Compound $\mathbf{3 b}$, $\mathbf{3 c}$ and $\mathbf{3 d}$ exhibited moderate activity.
\end{abstract}

Keywords: Benzofuran, Benzothiazol, Azetidinone, Antimicrobial activity.

\section{Introduction}

Benzofuran derivatives posses a wide range of biological activities. They have been reported to possess antimicrobial ${ }^{1-4}$, antitumour ${ }^{5,6}$, anti-inflammatory ${ }^{7}$ activity etc. Benzothiazoles play a significant role as antibacterial ${ }^{8-10}$ and antifungal activity. Azetidinones with heterocyclic molecule has created an excellent drug for antimicrobial ${ }^{11-13}$ activity. Based on these findings and in continuation of our research work, we report herein synthesis of a tri-heterocyclic molecule containing benzofuran, benzothiazole and azetidinone for enhanced activity.

\section{Experimental}

Melting points were determined in open capillary tube and are uncorrected. The TLC was taken in silica gel G precoated TLC plates. The solvents and reagents used for synthesis were of laboratory grade. The IR was recorded with Perkin Elmer FTIR spectrophotometer using $\mathrm{KBr}$ press pellet technique. The NMR was recorded in Bruker $400 \mathrm{MHz}$ FTNMR spectrometer using DMSO as solvent and TMS as internal standard. 


\section{Synthesis of 2-acetyl benzofuran (1)}

The mixture of salicylaldehyde ( 0.1 mole), chloroacetone $(0.1$ mole) and anhydrous potassium carbonate $(30 \mathrm{~g})$ were gently refluxed in dry acetone $(150 \mathrm{~mL})$ for $13 \mathrm{~h}$. The reaction product after cooling was filtered and the filtrate on the removal of the solvent under reduced pressure furnished 2-acetyl benzofuran as dark yellow colored solid. The product obtained was recrystallized from petroleum ether. Yield 55\% $\mathrm{IR} \mathrm{cm}^{-1}: 1674(\mathrm{C}=\mathrm{O}), 3088(\mathrm{CH}$ phenyl), 2900 $\left(\mathrm{CH}_{3}\right){ }^{1} \mathrm{H}$ NMR $\delta \mathrm{ppm} \mathrm{CHCl}{ }_{3}$ (400MHz): 7.46 to 7.73 aromatic protons, $2.2\left(3 \mathrm{H}, \mathrm{s}, \mathrm{CH}_{3}\right)$.

\section{Synthesis of 2- aminobenzothiazole}

To a solution of aniline $(0.2$ mole $)$ and potassium thiocyanate $(0.8$ mole $)$ in $360 \mathrm{~mL}$ of $96 \%$ acetic acid was added drop wise, with stirring, 0.2 mole of bromine dissolved in $250 \mathrm{~mL}$ of glacial acetic acid while the temperature was kept below $35{ }^{\circ} \mathrm{C}$. After all the bromine solution had been added the mixture was stirred for $10 \mathrm{~h}$ and was then filtered and the residue washed with water. The combined filtrate and washings were neutralized with ammonium hydroxide. The precipitate was collected on a filter and dried. Yield $70 \% \mathrm{IR} \mathrm{cm}^{-1}$ : $3056\left(\mathrm{CH}\right.$ aromatic), $3397(\mathrm{NH}){ }^{1} \mathrm{H}$ NMR $\delta \mathrm{ppm}(400 \mathrm{MHz}): 7.14$ to 7.6 aromatic protons, $5.55(1 \mathrm{H}, \mathrm{s}, \mathrm{NH})$.

\section{Synthesis of N-[(1Z)-1-(benzofuran-2-yl)ethylidene]1,3-benzothiazol-2-amine (2)}

A mixture of 2- acetyl benzofuran and 2-aminobenzothiazole and a small pinch of $p$-toluene sulphonic acid were gently refluxed in ethanol $(30 \mathrm{~mL})$ for $6 \mathrm{~h}$. After the completion of the reaction the mixture was poured into crushed ice, filtered and then dried and recrystallised from ethanol. Yield $60 \% \mathrm{IR} \mathrm{cm}^{-1}: 2929\left(\mathrm{CH}_{3}\right), 3058(\mathrm{CH}$ aromatic), 3119(CH aromatic), $1643(\mathrm{C}=\mathrm{N}){ }^{1} \mathrm{H} \mathrm{NMR} \delta \mathrm{ppm} \mathrm{CHCl}_{3}(400 \mathrm{MHz})$ aromatic protons 7.28 to $7.68,2.1(3 \mathrm{H}, \mathrm{s}, \mathrm{CH} 3)$.

Synthesis of 4-[1-benzofuran-2-yl]-1-[1,3-benzothiazol-2-yl]-3-azetidin-2-one (3a-3j) $N$-[(1Z)-1-(benzofuran-2-yl)ethylidene]1,3-benzothiazol-2-amine(2) (0.01 mole) was added to triethylamine $(0.02 \mathrm{~mol})$ in dioxane $(30 \mathrm{~mL})$ at 0 to $5{ }^{\circ} \mathrm{C}$. To this mixture various acid chloride $(0.012 \mathrm{~mol})$ was added drop wise. This mixture was stirred for $5 \mathrm{~h}$ at 0 to $5^{\circ} \mathrm{C}$. The precipitate formed was filtered off and the filtrate was refluxed for $6 \mathrm{~h}$. The reaction mixture was left for $24 \mathrm{~h}$ at room temperature and it was poured into crushed ice. The product (3a-3j) was precipitated, which was filtered, dried and recrystallised from ethanol.

Table 1. Physical data of synthesized compounds (3a-3j).

\begin{tabular}{cllcc}
\hline Compound & $\begin{array}{c}\text { Molecular formula \& } \\
\text { (Molecular weight) }\end{array}$ & \multicolumn{1}{c}{$\mathrm{R}$ (Acid chlorides) } & Yield, \% & M.P, ${ }^{\circ} \mathrm{C}$ \\
\hline 3a & $\mathrm{C}_{18} \mathrm{H}_{11} \mathrm{ClN}_{2} \mathrm{O}_{2} \mathrm{~S}(55)$ & $\mathrm{ClCH}_{2^{-}}$ & 55 & 220 \\
3b & $\mathrm{C}_{18} \mathrm{H}_{12} \mathrm{~N}_{2} \mathrm{O}_{2} \mathrm{~S}(320)$ & $\mathrm{CH}_{3^{-}}$ & 58 & 235 \\
3c & $\mathrm{C}_{19} \mathrm{H}_{14} \mathrm{~N}_{2} \mathrm{O}_{2} \mathrm{~S}(334)$ & $\mathrm{CH}_{3} \mathrm{CH}_{2-}$ & 63 & 240 \\
3d & $\mathrm{C}_{19} \mathrm{H}_{13} \mathrm{BrN}_{2} \mathrm{O}_{2} \mathrm{~S}(413)$ & $\mathrm{BrCH}_{2^{-}}$ & 53 & 210 \\
3e & $\mathrm{C}_{18} \mathrm{H}_{10} \mathrm{Cl}_{2} \mathrm{~N}_{2} \mathrm{O}_{2} \mathrm{~S}(389)$ & $\mathrm{Cl}_{2} \mathrm{CH}-$ & 54 & 215 \\
$\mathbf{3 f}$ & $\mathrm{C}_{21} \mathrm{H}_{18} \mathrm{~N}_{2} \mathrm{O}_{2} \mathrm{~S}(362)$ & Pentanoyl chloride & 50 & 225 \\
$\mathbf{3 g}$ & $\mathrm{C}_{20} \mathrm{H}_{10} \mathrm{Cl}_{2} \mathrm{~N}_{2} \mathrm{O}_{4} \mathrm{~S}(445)$ & Oxalylacetyl chloride & 40 & 240 \\
$\mathbf{3 h}$ & $\mathrm{C}_{20} \mathrm{H}_{13} \mathrm{ClN}_{2} \mathrm{O}_{3} \mathrm{~S}(396)$ & Succinyl chloride & 42 & 245 \\
$\mathbf{3 i}$ & $\mathrm{C}_{20} \mathrm{H}_{16} \mathrm{~N}_{2} \mathrm{O}_{2} \mathrm{~S}(48)$ & $n$-Butyryl chloride & 44 & 243 \\
3j & $\mathrm{C}_{19} \mathrm{H}_{14} \mathrm{~N}_{2} \mathrm{O}_{3} \mathrm{~S}(350)$ & $\begin{array}{l}\text { Methoxy acetyl } \\
\text { chloride }\end{array}$ & 47 & 230 \\
\hline
\end{tabular}

All compounds gave satisfactory $\mathrm{CH}$ and $\mathrm{N}$ analysis. 


\section{Spectral values}

4-(1-Benzofuran-2-yl)-1-(1,3-benzothiazol-2-yl)-3-chloro-4-methylazetidin-2one (3a)

IR cm ${ }^{-1}: 2926\left(\mathrm{CH}_{3}\right), 3057(\mathrm{CH} \mathrm{Ar}), 3119(\mathrm{CH} \mathrm{Ar}), 743(\mathrm{CCl}), 1673$ (CO cyclic amide) ${ }^{1} \mathrm{H}$ NMR $\delta$ ppm $\mathrm{CHCl}_{3}: 2.61\left(3 \mathrm{H}, \mathrm{s}, \mathrm{CH}_{3}\right), 4.56(1 \mathrm{H}, \mathrm{s}$, Azetidinone), $7.16(1 \mathrm{H}, \mathrm{s}, \mathrm{CH}), 7.31$ to 7.71 $(8 \mathrm{H}, \mathrm{m}, \mathrm{Ar})$.

4-(1-Benzofuran-2-yl)-1-(1, 3-benzothiazol-2-yl)-4-methylazetidin-2-one (3b)

IR cm ${ }^{-1}: 2772\left(\mathrm{CH}_{2}\right), 2958\left(\mathrm{CH}_{3}\right), 3057(\mathrm{CH} \mathrm{Ar}), 3072(\mathrm{CH} \mathrm{Ar}), 1695$ (CO cyclic amide) ${ }^{1} \mathrm{H}$ NMR $\delta$ ppm $\mathrm{CHCl}_{3}: 2.28\left(3 \mathrm{H}, \mathrm{s}, \mathrm{CH}_{3}\right), 2.61\left(2 \mathrm{H}, \mathrm{s}, \mathrm{CH}_{2}\right), 7.26(1 \mathrm{H}, \mathrm{s}, \mathrm{CH}), 7.33$ to 7.86 $(8 \mathrm{H}, \mathrm{m}, \mathrm{Ar})$.

4-(1-Benzofuran-2-yl)-1-(1,3-benzothiazol-2-yl)-3,4-dimethylazetidin-2-one (3c)

IR cm ${ }^{-1}: 2938\left(\mathrm{CH}_{3}\right), 2975(\mathrm{CH} \mathrm{Ar}), 3043(\mathrm{CH} \mathrm{Ar}), 1682$ (CO cyclic amide) $2734(\mathrm{CH}$, Azetidinone), ${ }^{1} \mathrm{H}$ NMR $\delta \mathrm{ppm} \mathrm{CHCl}_{3}: 1.24\left(3 \mathrm{H}, \mathrm{d}, \mathrm{CH}_{3}\right), 2.61\left(3 \mathrm{H}, \mathrm{s}, \mathrm{CH}_{3}\right), 7.26(1 \mathrm{H}, \mathrm{s}, \mathrm{CH})$, 7.31to7.71 $(8 \mathrm{H}, \mathrm{m}, \mathrm{Ar}), 2.52\left(1 \mathrm{H}, \mathrm{q}, \mathrm{CH}\right.$ Azetidinone), ESI MS $(\mathrm{m} / \mathrm{z})=\mathrm{M}+\mathrm{H}=349, \mathrm{M}^{+}=$ 348 , fragmented ion at 215 and 216.

4-(1-Benzofuran-2-yl)-1-(1,3-benzothiazol-2-yl)-3-bromo-4-methylazetidin-2-one (3d)

IR cm ${ }^{-1}$ : $2850(\mathrm{CH}), 2908\left(\mathrm{CH}_{3}\right), 2957$ (CH Ar), 3055 (CH Ar), $754(\mathrm{CBr}), 1637$ (CO cyclic amide) ${ }^{1} \mathrm{H}$ NMR $\delta$ ppm $\mathrm{CHCl}_{3}: 1.41\left(3 \mathrm{H}, \mathrm{s}, \mathrm{CH}_{3}\right), 4.48(1 \mathrm{H}, \mathrm{s}, \mathrm{CH}), 7.13(1 \mathrm{H}, \mathrm{s}, \mathrm{CH}), 7.15$ to 7.69 $(8 \mathrm{H}, \mathrm{m}, \mathrm{Ar}) \operatorname{ESI} \mathrm{MS}(\mathrm{m} / \mathrm{z})=\mathrm{M}+\mathrm{H}=414, \mathrm{M}^{+}=413$, fragmented ion at 285.

4-(1-Benzofuran-2-yl)-1-(1,3-benzothiazol-2-yl)-3,3-dichloro-4-methylazetidin-2one $(3 e)$

IR cm ${ }^{-1}: 2850\left(\mathrm{CH}_{3}\right), 2918(\mathrm{CH} \mathrm{Ar}), 3059(\mathrm{CH} \mathrm{Ar}), 754(\mathrm{CCl}), 1696\left(\mathrm{CO}\right.$ cyclic amide) ${ }^{1} \mathrm{H}$ NMR $\delta$ ppm $\mathrm{CHCl}_{3}: 1.18\left(3 \mathrm{H}, \mathrm{s}, \mathrm{CH}_{3}\right), 7.19(1 \mathrm{H}, \mathrm{s}, \mathrm{CH}), 7.23$ to $7.78(8 \mathrm{H}, \mathrm{m}, \mathrm{Ar})$.

\section{Antimicrobial activity}

The minimum inhibitory concentration was determined by the method of serial dilutions and found to be $100 \mu \mathrm{g} / \mathrm{mL}$ for bacteria and $200 \mu \mathrm{g} / \mathrm{mL}$ for fungi. The newly synthesized compounds were screened for antibacterial activity using cup plate diffusion method; $B$. subtilis and E. coli were taken as test organisms. Among the tested compounds $\mathbf{3 b}, \mathbf{3 c}$ and $\mathbf{3 d}$ were moderately active against standard Ampicillin while the remaining compounds were weakly active. The fungicidal activity of the compounds was evaluated against $C$. albicans using griseofulvin as standard, compounds $\mathbf{3 b}, \mathbf{3 c}$ and $\mathbf{3 d}$ exhibited moderate activity.

Table 2. Antimicrobial activity of benzofuran derivatives (3a-3j)

\begin{tabular}{cccc}
\hline Compound code & $\begin{array}{c}\text { Bacillus subtilus } \\
\text { Zone of }\end{array}$ & $\begin{array}{c}\text { Escherichia coli } \\
\text { inhibition, diameter in mm }\end{array}$ & $\begin{array}{c}\text { Candida albicans } \\
\text { 3a }\end{array}$ \\
\hline 3b & 15 & 7 & 5 \\
3c & 17 & 14 & 9 \\
3d & 16 & 16 & 12 \\
3e & 11 & 15 & 11 \\
Ampicillin & 20 & 8 & 2 \\
Control & .04 & 20 & \\
Griseofulvin & - & .03 & 17 \\
Control & - & - & .04 \\
\hline
\end{tabular}




\section{Results and Discussion}

The scheme of synthesis of titled compounds is depicted in the (Scheme 1). All the intermediate and targeted molecules were confirmed by TLC, IR, NMR and mass data. 2acetyl benzofuran was synthesized by reacting salicylaldehyde and chloroacetone in presence of anhydrous potassium carbonate as reported. 2-Aminobenzothiazole was prepared by stirring aniline and potassium thiocyanate in presence of bromine and acetic acid in cold condition. $N$-[(1Z)-1-(benzofuran-2-yl)ethylidene]1,3-benzothiazol-2-amine(2) was obtained by refluxing 2-acetyl benzofuran and 2-aminobenzothiazole in presence of $p$ toluene sulphonic acid. The IR spectra of compound (2) showed presence of $\mathrm{C}=\mathrm{N}$ stretching vibrations at $1643 \mathrm{~cm}^{-1}$ and disappearance of $\mathrm{NH}$ absorption bands. The targeted molecule 4[1-benzofuran-2-yl]-1-[1,3-benzothiazol-2-yl]-3-bromo-4-methylazetidin-2-one (3d) was synthesized by mixing compound (2) with triethylamine in dioxane and bromoacetyl chloride in cold condition. The IR spectra revealed the peak at $1637 \mathrm{~cm}^{-1}$ showing the presence of $\mathrm{C}=\mathrm{O}$ stretching vibration of cyclic amide. The $\mathrm{C}-\mathrm{H}$ stretching frequency was at $2850 \mathrm{~cm}^{-1}$ and $\mathrm{C}-\mathrm{Br}$ stretching frequency at $754 \mathrm{~cm}^{-1}$ indicated their presence in azetidinone ring.

From ${ }^{1} \mathrm{H}$ NMR spectra $\delta$ value 1.41 was assigned to methyl protons, a singlet at $\delta$ value 4.48 was integrated to methine of azetidinone and the multiplet $\delta$ value at 7.15 to 7.69 was due to aromatic protons. The structure of compound (3d) was further supported by its ESI MS Spectra indicating molecular ion peak was at $413 \mathrm{~m} / \mathrm{z}$ and fragmented ion peak at $285 \mathrm{~m} / \mathrm{z}$.

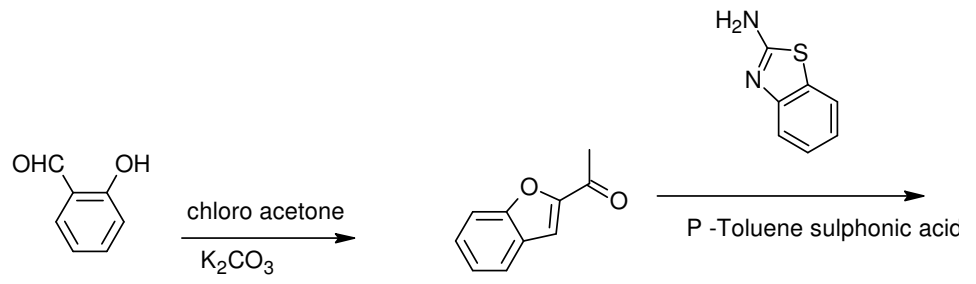

(1)

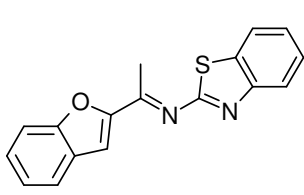

(2)

\section{Conclusion}

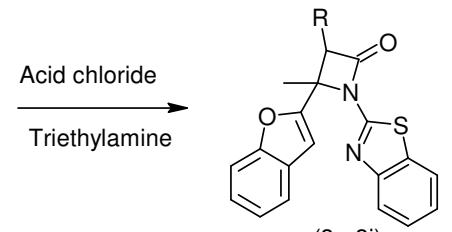

$$
\mathrm{R}=\mathrm{Br}-\mathrm{Cl}-\mathrm{CH}_{3}-\mathrm{CH}_{3} \mathrm{CH}_{2}-\text { etc. }
$$

\section{Scheme 1}

Various tri-heterocyclic compounds $\mathbf{3 a}$ to $\mathbf{3 j}$ of benzofuran derivatives were synthesized containing benzofuran, benzothiazol and azetidinone rings. The compounds were characterized by IR, NMR and ESI MS spectral data. They were evaluated for antimicrobial activity, compounds $\mathbf{3 b}, \mathbf{3} \mathbf{c}$ and $\mathbf{3 d}$ exhibited moderate activity when compared to standard Ampicillin and Griseofulvin

\section{Acknowledgment}

We are thankful to Chairman, Secretary and Principal of Krupanidhi College of Pharmacy Bangalore for encouragement and providing facilities and IISc, Bangalore. 


\section{References}

1. Manna K and Agarwal Y K, Bioorg Med Chem Lett., 2009, 19(10), 2688- 2692.

2. Alejandro Urzúa , Marcos C. Rezende, Carolina Mascayano and Loretta Vásquez, Molecules, 2008, 13(10), 882-891.

3. Aruna Kumar D B, Prakash G K, Kumarasamy M N, Nandheswarappa B P, Sheringara B S and Mahadevan K M, Indian J Chem., 2007, 46B, 336- 343.

4. Nalan Gundogdu K, Kadriya B, Yagmur T, Umit U and Seref D, Eur J Med Chem., 2006, 41, 6516

5. Hayakawa I, Shioya R, Agatsuma T, Furukawa H, Naruto S and Sugano Y,Bioorg Med Chem Lett., 2004, 14, 455- 458.

6. Galal S A, Abd El All, Abdullah M M and EL- Diwani HI, Bioorg Med Chem Lett., 2009, 19(9), 2420- 2428.

7. Lourdes Santana, Marta Teijeira, Eugenio Uriarte, Carmen Teran, Belen Liñares, Rosa Villar, Reyes Laguna and Ernesto Cano, Eur J Pharm Sci., 1999, 7, 161-166

8. Nargund LVG, Gurupadayya B M, Nagappa A N, Shivakumar B and Jayachandran E, Indian J Heterocycl Chem., 1998, 7, 213- 216.

9. Rajeeva B, Srinivasulu and Shantakumar, E Journal of Chemistry, 2009, 6(3), 775-779.

10. Iro A, Athina G, Paola V and Franca Z, ARKIVOC, 2009, (VI), 89-102.

11. Vijay Kumar.M M J, Jayadevaiah K V, Nagaraja T S, Bharathi D R, Shameer H, Jayachandran E and Sreenivasa G M, Arch Pharm Sci Res., 2009, 1(1), 31-39.

12. Govindarajan R, Jameela H J and Bhat A R, Indian J Heterocycl Chem., 2003, 12, 229- 232.

13. Joshi H S, Popat K H and Nimavt K S, Indian J Heterocycl Chem., 2003, 12, 225-228. 


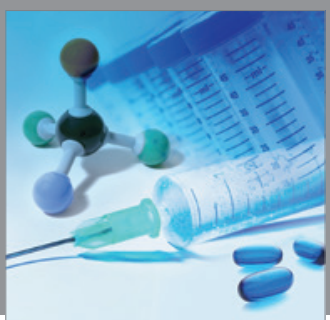

International Journal of

Medicinal Chemistry

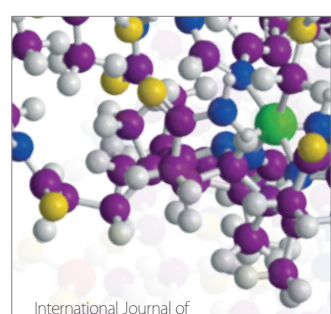

Carbohydrate Chemistry

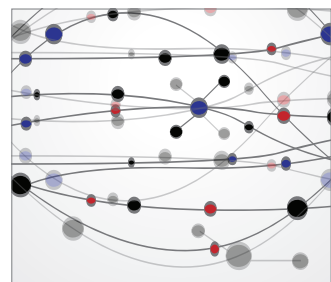

The Scientific World Journal
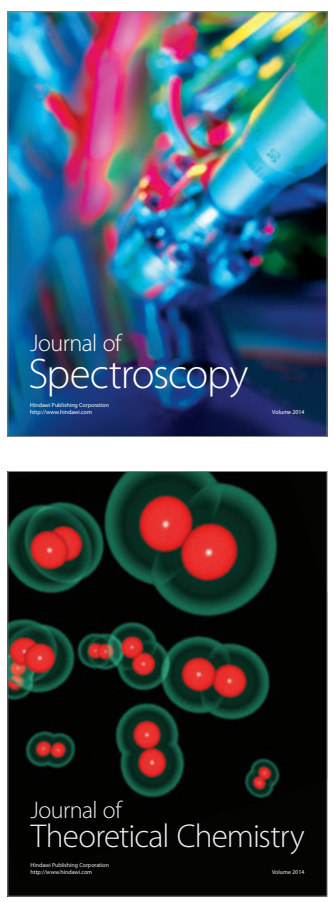
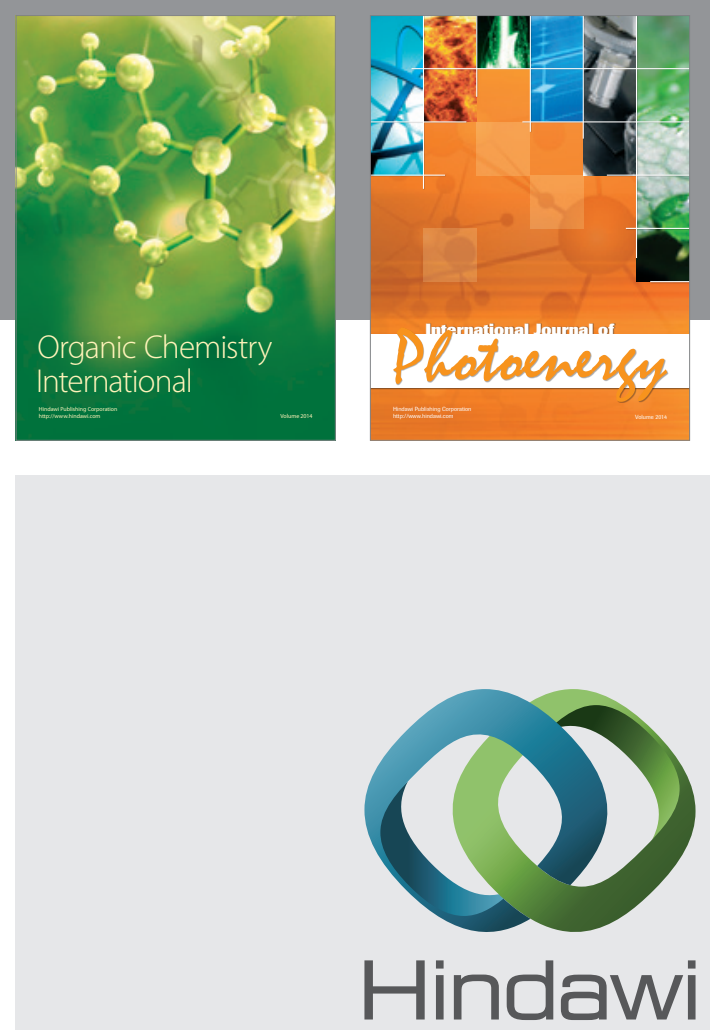

Submit your manuscripts at

http://www.hindawi.com
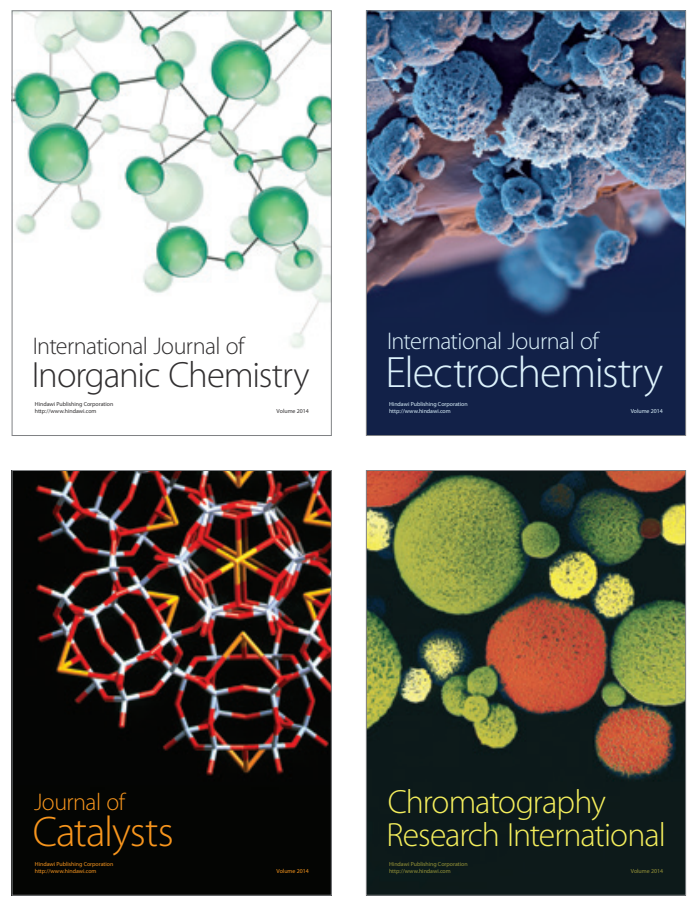
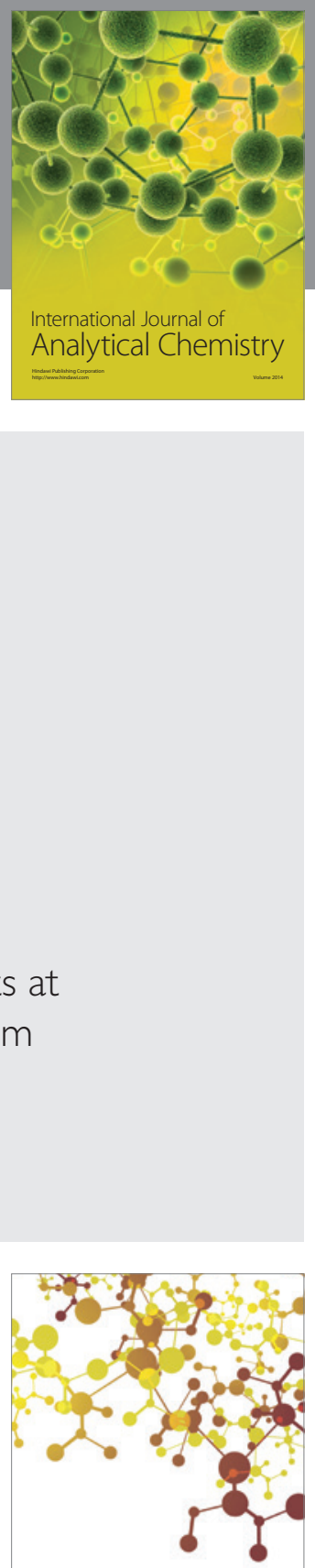

Journal of

Applied Chemistry
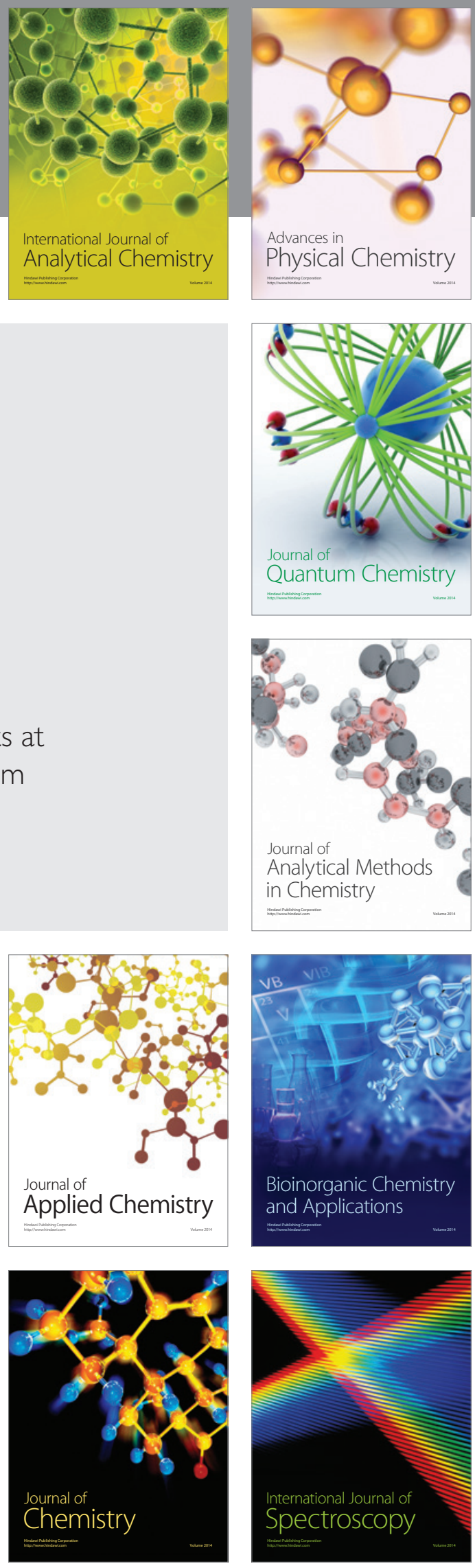